\title{
A Decision Support System is Needed for Rapid Triage of Chest Pain Patients Using High Sensitivity Troponin Testing-Based Algorithms
}

\author{
Chien-Chang Lee, MD, ScD' ${ }^{*}$; Cheng-Heng Liu, MD'; Sih-Shiang Huang, MD²; Cho-Han Chiang² \\ 'Department of Emergency Medicine, National Taiwan University, Taipei 106, Taiwan \\ ${ }^{2}$ Department of Medicine, College of Medicine, National Taiwan University, Taipei, Taiwan

\section{${ }^{*}$ Corresponding author} \\ Chien-Chang Lee, MD, ScD \\ Director, Department of Emergency Medicine, National Taiwan University Hospital, No. 7, Chung-Shan South Road, Taipei 100, Taiwan; Tel. +886-2-23 I23456 ext \\ 6283I;
}

\section{Article information}

Received: March 21 ${ }^{\text {st }}, 2019 ;$ Accepted: March 25 $5^{\text {th }}, 2019$; Published: April 2 ${ }^{\text {nd }}, 2019$

\section{Cite this article}

Lee C-C, Liu C-H, Huang S-S, Chiang C-H.A decision support system is needed for rapid triage of chest pain patients using high sensitivity troponin testing-based algorithms. Emerg Med Open J. 2019; 5(I): el-e2. doi: I0. I7I40/EMOJ-5-e006

$\mathrm{C}$ ardiovascular disease is a leading cause of mortality and morbidity worldwide. In 2013, an estimated 8.14 million people died from acute myocardial infarction (AMI) globally. ${ }^{1}$ Emergency department (ED) is the main portal of entry for patients with acute chest pain symptoms. Approximately $10 \%$ of all emergency department (ED) patients presented with chest pain complaint. ${ }^{2}$ However, only $13-23.6 \%$ of these patients are finally diagnosed with AMI. ${ }^{3}$ Patients with segment (ST) elevation MI (STEMI) could be rapidly recognized by electrocardiogram (ECG) exam, but patients with non-ST elevation MI (NSTEMI) could not be efficiently excluded or included by contemporary cardiac troponin assays. Contemporary troponin assay could not detect the presence of cardiac troponins in the peripheral circulation until 6 to 8 hours after the onset of MI. ${ }^{4}$ Therefore, patients presenting to the ED with chest pain usually require serial troponin measurements over 6 to 8 hours before the possibility of MI can be safely excluded. Failure in early detection results in higher rate of complications and mortality. The delay in ruling out MI constitutes a major cause of ED crowding, and the delay in ruling in MI may lead to poor prognosis. It has been shown early evidence-based management, including antiplatelet treatment and revascularization, may decrease mortality, shorten hospitalization, and improve overall outcome. ${ }^{5}$

The introduction of high sensitive cardiac troponin (hscTn) assays in 2010 has shown promise to enhance the accuracy and efficiency of MI diagnosis in the ED tremendously. ${ }^{6}$ These assays measure $\mathrm{c}^{\mathrm{T}} \mathrm{n}$ in the single digit range of nanograms per liter, about 10- to 100-fold lower than the contemporary c'Tn assays. ${ }^{7}$ This has led to the development of several hs-cTn-based accelerated diagnostic algorithms to triage patients with suspected acute coronary syndromes (ACS) efficiently and safely. ${ }^{6}$ Several accelerated diag- nosis algorithms, such as 0-, 1-, or 2-hour algorithm, have been developed and implemented based on the high sensitivity nature of new generation troponins. ${ }^{8-10}$ One such promising algorithm is the 0/1-hour algorithm recommended by the European Society of Cardiology (ESC). ${ }^{11}$ This algorithm combines assay-specific cutoff values of hs-cTn at presentation and the absolute 1-hour changes (0-1 delta) to rapidly rule out or rule in patients with NSTEMI. Thus, clinical decision could be made for patients who presented to the ED with suspected AMI or ACS in just 2 to 3 hours after ED presentation. Although concern has been raised regarding the safety of the $0 / 1$-hour algorithm in patients with renal dysfunction, in a recent work, Twerenbold $\mathrm{R}$ showed the $0 / 1$-hour algorithms using either high-sensitivity cardiac troponin $\mathrm{T}$ or troponin I can rule out MI rapidly and safely. ${ }^{12}$ In addition, recent studies have shown combining HEART score or combining hs-c'Tn'T and hs-c'TnI may further enhance the accuracy of the rapid diagnosis algorithm. ${ }^{13,14}$

Despite the rapid development of various high sensitivity troponin testing based accelerated diagnosis system, the implementation of the newly developed algorithms in ED have been slow. One of the big obstacle is the complexity of the algorithms that include several cutoff values of troponin at several time points. The addition of scoring systems and multiple markers strategy further complicates the decision process. Because the hospital central laboratory conventionally reports only the test results with reference value, an emergency physician therefore, has to memorize the complex algorithms. The 1-hour algorithm is a very good example that a clinical decision support system will help clinicians make evidence-supported decisions. A decision support system will help remind the various time points for blood sampling and also pro- 
vide reference cutoff values of troponin tests at different time points. ${ }^{15}$ In addition, the system can also remind the physician with the update prognosis data in different decision groups and automatically calculates TIMI, GRACE or HEART scores. Up to date, such system is not commercially available, but the design and implementation of such system in the laboratory information system is not technically difficult. We call for the design of such a system to help emergency physicians make evidence-based decision in the era of information tsunami.

\section{CONFLICTS OF INTEREST}

The authors declare that they have no conflicts of interest.

\section{REFERENCES}

1. Roth GA, Huffman MD, Moran AE, et al. Global and regional patterns in cardiovascular mortality from 1990 to 2013. Circulation. 2015; 132(17): 1667-1678. doi: 10.1161/CIRCULATIONAHA. 114.008720

2. Goodacre S, Cross E, Arnold J, Angelini K, Capewell S, Nicholl J. The health care burden of acute chest pain. Heart. 2005; 91(2): 229-230. doi: 10.1136/hrt.2003.027599

3. Bhuiya FA, Pitts SR, McCaig L. Emergency department visits for chest pain and abdominal pain: United States, 1999-2008. NCHS data brief. 2010; (43): 1-8.

4. Macrae AR, Kavsak PA, Lustig V, et al. Assessing the requirement for the 6-hour interval between specimens in the American Heart Association Classification of Myocardial Infarction in Epidemiology and Clinical Research Studies. Clin Chem. 2006; 52(5): 812-818. doi: 10.1373/clinchem.2005.059550

5. Neumann FJ, Sousa-Uva M, Ahlsson A, et al. 2018 ESC/ EACTS guidelines on myocardial revascularization. Eur Heart J. 2018, 40(2): 87-165. doi: 10.1093/eurheartj/ehy394

6. Twerenbold R, Boeddinghaus J, Nestelberger T, et al. Clinical use of high-sensitivity cardiac troponin in patients with suspected myocardial infarction. J Am Coll Cardiol. 2017; 70(8): 996-1012. doi: 10.1016/j.jacc.2017.07.718

7. Garg P, Morris P, Fazlanie AL, et al. Cardiac biomarkers of acute coronary syndrome: From history to high-sensitivity cardi- ac troponin. Intern Emerg Med. 2017; 12(2): 147-155. doi: 10.1007/ s11739-017-1612-1

8. Body R, Carley SD, McDowell G, et al. Use of low level high sensitivity troponin to rule out acute myocardial infarction in the emergency department. European Heart Journal. Supplement 2010; 12: F111-F112.

9. Reichlin T, Schindler C, Drexler B, et al. One-hour rule-out and rule-in of acute myocardial infarction using high-sensitivity cardiac troponin T. Arch Intern Med. 2012; 172(16): 1211-1218. doi: $10.1001 /$ archinternmed.2012.3698

10. Reichlin T, Cullen L, Parsonage WA, et al. Two-hour algorithm for triage toward rule-out and rule-in of acute myocardial infarction using high-sensitivity cardiac troponin T. Am J Med. 2015; 128(4): 369-379.e364. doi: 10.1016/j.amjmed.2014.10.032

11. Roffi M, Patrono C, Collet JP, et al. 2015 ESC Guidelines for the management of acute coronary syndromes in patients presenting without persistent ST-segment elevation: Task force for the management of acute coronary syndromes in patients presenting without persistent ST-segment elevation of the European Society of Cardiology (ESC). Eur Heart J. 2016; 37(3): 267-315. doi: 10.1093/eurheartj/ehv320

12. Twerenbold R, Badertscher P, Boeddinghaus J, et al. 0/1-Hour triage algorithm for myocardial infarction in patients with renal dysfunction. Circulation. 2018; 137(5): 436-451. doi: 10.1161/CIRCULATIONAHA.117.028901

13. van der Linden N, Wildi K, Twerenbold R, et al. Combining high-sensitivity cardiac troponin $\mathrm{I}$ and cardiac troponin $\mathrm{T}$ in the early diagnosis of acute myocardial infarction. Circulation. 2018; 138(10): 989-999. doi: 10.1161/CIRCULATIONAHA.117.032003

14. Ljung L, Lindahl B, Eggers KM, et al. A rule-out strategy based on high-sensitivity troponin and HEART score reduces hospital admissions. Ann Emerg Med. 2019; pii: S0196-0644(18)31529-4. doi: 10.1016/j.annemergmed.2018.11.039

15. Castaneda C, Nalley K, Mannion C, et al. Clinical decision support systems for improving diagnostic accuracy and achieving precision medicine. J Clin Bioinforma. 2015; 5: 4. doi: 10.1186/s13336015-0019-3 